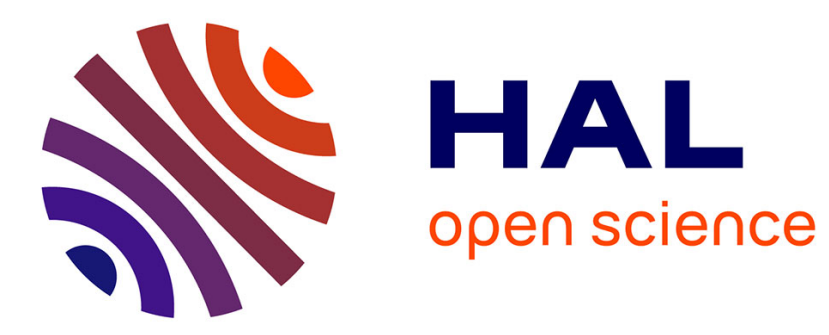

\title{
Data Fusion-Based Descriptor Approach for Attitude Estimation under accelerated maneuvers
}

\author{
Aida Makni, Alain Kibangou, Hassen Fourati
}

\section{To cite this version:}

Aida Makni, Alain Kibangou, Hassen Fourati. Data Fusion-Based Descriptor Approach for Attitude Estimation under accelerated maneuvers. Asian Journal of Control, 2019, 21 (4), pp.1433-1442. 10.1002/asjc.2084 . hal-01982463

\section{HAL Id: hal-01982463 https://hal.science/hal-01982463}

Submitted on 26 Feb 2019

HAL is a multi-disciplinary open access archive for the deposit and dissemination of scientific research documents, whether they are published or not. The documents may come from teaching and research institutions in France or abroad, or from public or private research centers.
L'archive ouverte pluridisciplinaire HAL, est destinée au dépôt et à la diffusion de documents scientifiques de niveau recherche, publiés ou non, émanant des établissements d'enseignement et de recherche français ou étrangers, des laboratoires publics ou privés. 


\title{
Data Fusion-Based Descriptor Approach for Attitude Estimation under accelerated maneuvers
}

\author{
Aida Makni, Alain Y. Kibangou, and Hassen Fourati
}

\begin{abstract}
This paper proposes the design of an attitude estimation algorithm for a rigid body subject to accelerated maneuvers. Unlike the current literature where the process model is usually driven by triaxial gyroscope measurements, we investigate a new formulation of the state-space model where the process model is given by triaxial accelerometer measurements. The observation model is given by triaxial gyroscope and magnetometer measurements. The proposed model is written as a descriptor system and takes the external acceleration sensed by the accelerometer into account. Based on this model, a Quaternion Descriptor Filter (QDF) is developped and its performance is evaluated through simulations and experimental tests in pedestrian navigation.
\end{abstract}

Key Words: Attitude estimation, quaternion, descriptor filter, MARG sensors, external acceleration.

\section{Introduction}

Attitude estimation of rigid bodies is a prerequisite for successful navigation in a wide range of applications including robotics [1], satellite control [2] and smartphone applications [3]. Attitude estimation is usually achieved with magnetic, angular rate, and gravity (MARG) sensors [4], [5]. MARG sensors usually contain a 3-axis magnetometer, a 3-axis gyroscope, and a 3-axis accelerometer which measure the Earth's magnetic field, the angular velocity, and the sum of external acceleration and gravity of rigid body, respectively, when it is under motion [6]. These sensors are often cheap and suffer from high noise level and bias. Hence it is necessary to derive efficient fusion methods to get accurate attitude despite sensors inaccuracies. For this purpose, an abundant literature can be found with methods including Kalman filters $[7,8]$, complementary filters $[9,10]$, and

\footnotetext{
A. Makni, Alain Y. Kibangou and H. Fourati, are with Univ. Grenoble Alpes, CNRS, Inria, Grenoble INP, GIPSA-Lab, 38000 Grenoble, France. (e-mails: aidamakni@gmail.com, alain.kibangoulgipsa-lab.fr,

hassen.fourati@gipsa-lab.fr).
}

nonlinear observers $[11,12]$. The attitude of a rigidbody can be represented by rotation matrices, Euler angles, or a quaternion. The latter case gives rise to a state-space model with quaternion as system state, while the process model is derived from the attitude kinematic equation, which depends on angular velocity measurements provided by the gyroscope. The observation model is built from accelerometer and magnetometer measurements. When the accelerometer measures gravity, the above observation model is enough to provide a reliable attitude. However, when the external acceleration is large due to high-acceleration maneuvers, the accelerometer output represents the sum of gravity and external acceleration. In this case, the accelerometer is no longer able to estimate the gravity direction yielding an erroneous attitude estimate.

To deal with the impact of external acceleration on attitude estimation methods, two main approaches are usually considered in the literature. The first one is purely software-oriented whereas the second one needs additional hardware (GNSS receptors). The first approach follows the detect and correct paradigm, where correction is usually obtained by giving small weights to accelerometer measurements in the data fusion process $[1,13,14]$. Detecting the external 
acceleration can be carried out by setting-up some thresholds [13, 15], or directly by estimating the external acceleration for short accelerated periods [14]. In [15], an estimation method based on adaptive filtering theory is proposed; the covariance matrix of the accelerometer noise is tuned according to the filter residual. The underlying assumption is that the external acceleration is of short duration, which is not always true. The main idea of the second approach, known as velocity-aided estimation, is to augment the available measurements with the linear velocity provided by GPS/GNSS devices [16]. Although they have been successfully used in several applications, velocity aided systems lose performance when GPS data are no longer available, as in indoor applications.

In this paper, we introduce a third approach with the following features: (i) it is fully model-oriented, (ii) it complies with external accelerations of any duration and magnitude in the case of pedestrian navigation, (iii) it does not need GPS measurements, (iv) it makes use of accelerometer measurements to build the process model instead of gyroscope measurements. Hence, the resulting model is singular and can be analyzed through the framework of descriptor systems. In this modeling approach, both dynamics and constraints on the variables are taken into account. State estimation for descriptor systems has been studied several years ago and is still a promising research area. Some recent works include [17, 18].

The paper is organized as follows: Some notations and definitions are stated in Section II before deriving the new model and the corresponding filter in Section III. Then an evaluation of the proposed approach is carried out in Section IV by means of numerical simulations and comparisons with relevent methods. Experimental tests under high accelerations scenario and agressive movements are proposed in Section V before concluding the paper.

\section{Background: quaternion algebra and sensor measurement models}

The rigid body attitude in 3D motion is determined when the orientation axes of the body frame $B\left(X_{B}, Y_{B}, Z_{B}\right)$ are specified with respect to a local Earth-fixed frame $N\left(X_{N}, Y_{N}, Z_{N}\right)$. The $X_{N}$ (resp. $Z_{N}$ )-axis points towards the North (resp. the Earth's interior perpendicularly). The $Y_{N}$-axis completes the right-handed coordinate system, pointing East (NED: North, East, Down). The attitude and the altitude are two decoupled variables and thus the change of attitude is not affected by the altitude variation.
In this paper, the unit norm quaternion is used to represent the attitude. It is defined as:

$$
q=\left[\begin{array}{ll}
q_{0} & \vec{q}^{T}
\end{array}\right]^{T}=\left[\begin{array}{llll}
q_{0} & q_{1} & q_{2} & q_{3}
\end{array}\right]^{T} \in \mathbb{R}^{4},
$$

where $q_{0}$ and $\vec{q}$ are the scalar and the vector parts of the quaternion, respectively. The rotation matrix between $N$ and $B$ is given by:

$C(q)=\left(\begin{array}{lll}2 q_{0}^{2}+2 q_{1}^{2}-1 & 2 q_{1} q_{2}+2 q_{0} q_{3} & 2 q_{1} q_{3}-2 q_{0} q_{2} \\ 2 q_{1} q_{2}-2 q_{0} q_{3} & 2 q_{0}^{2}+2 q_{2}^{2}-1 & 2 q_{2} q_{3}+2 q_{0} q_{1} \\ 2 q_{1} q_{3}+2 q_{0} q_{2} & 2 q_{2} q_{3}-2 q_{0} q_{1} & 2 q_{0}^{2}+2 q_{3}^{2}-1\end{array}\right)$.

In this paper, the sensor configuration for attitude estimation consists of a 3-axis gyroscope, a 3axis accelerometer and a 3-axis magnetometer. As commonly adopted [19], their outputs are respectively given by:

$$
\begin{aligned}
y_{g} & =\omega+b_{g}+\delta_{g}, \\
y_{a} & =C(q)\left(G+a_{p}\right)+\delta_{a}, \\
y_{m} & =C(q) m+\delta_{m},
\end{aligned}
$$

where $y_{g}, y_{a}$ and $y_{m} \in \mathbb{R}^{3}$ denote the outputs expressed in $B, \omega=\left[\begin{array}{lll}\omega_{x} & \omega_{y} & \omega_{z}\end{array}\right]^{T}$ and $b_{g}$ are respectively the true angular velocity and the gyroscope bias expressed in $B, a_{p}=\left[\begin{array}{lll}a_{p x} & a_{p y} & a_{p z}\end{array}\right]^{T}$ denotes the persistent external acceleration vector (nongravitational acceleration), $G=\left[\begin{array}{lll}0 & 0 & g\end{array}\right]^{T}$ is the gravity vector $\left(g=9.81 \mathrm{~m} / \mathrm{s}^{2}\right), m=\left[\begin{array}{ccc}m_{x} & m_{y} & m_{z}\end{array}\right]^{T}$ represents the theoretical components of Earth's magnetic field measured in $N$. Actually, the parameters of the theoretical geomagnetic field $m$ depends on the location on Earth [20]. The accelerometer drift is usually negligible and is not considered in this paper.

Remark II.1 The static bias of the gyroscope $b_{g}$, is a slow-varying signal. In practice, it can be easily estimated by taking the long term average of the measurements when the gyroscope is not subject to any motion. Therefore, we neglect the bias term in the filter design but in the simulation and experimental sections, we subtract it from the measurements. As a consequence, the gyroscope output in Eq.(3), can be rewritten as:

$$
y_{g}=\omega+\delta_{g} .
$$

In the sequel, we adopt the following assumption and notations:

Assumption II.2 $\delta_{g}, \delta_{a}$, and $\delta_{m}$ are assumed to be mutually independent and identically distributed with known covariance matrices $R_{g}=\sigma_{g}^{2} I_{3}, R_{a}=\sigma_{a}^{2} I_{3}$, and $R_{m}=\sigma_{m}^{2} I_{3}$, respectively. $I_{3}$ being the $3 \times 3$ identity matrix. 
We denote by $\left[x^{\times}\right]$the skew matrix associated with a vector $x=\left[\begin{array}{lll}x_{1} & x_{2} & x_{3}\end{array}\right]^{T} \in \mathbb{R}^{3}$ :

$$
\left[x^{\times}\right]=\left(\begin{array}{ccc}
0 & -x_{3} & x_{2} \\
x_{3} & 0 & -x_{1} \\
-x_{2} & x_{1} & 0
\end{array}\right) .
$$

The quaternion product $\otimes$ of two unit-norm quaternions $q_{a}=\left[\begin{array}{ll}q_{a 0} & \overrightarrow{q a}^{T}\end{array}\right]^{T}$ and $q_{b}=\left[\begin{array}{ll}q_{b 0} & \overrightarrow{q b}^{T}\end{array}\right]^{T}$ is defined as:

$$
q_{a} \otimes q_{b}=\left(\begin{array}{cc}
q_{a 0} & -{\overrightarrow{q_{a}}}^{T} \\
\overrightarrow{q_{a}} & q_{a 0} I_{3}+\left[{\overrightarrow{q_{a}}}^{\times}\right]
\end{array}\right)\left(\begin{array}{c}
q_{b 0} \\
\overrightarrow{q_{b}}
\end{array}\right),
$$

We consider two vectors $b$ and $r$ in $\mathbb{R}^{3}$, and their associated quaternions $b_{q}=\left[\begin{array}{ll}0 & b^{T}\end{array}\right]^{T}$ and $r_{q}=$ $\left[\begin{array}{ll}0 & r^{T}\end{array}\right]^{T}$ in $\mathbb{R}^{4}$. The quaternion product can be expressed as:

$$
\begin{aligned}
q \otimes b_{q} & =\Omega(b) q, \\
r_{q} \otimes q & =\Upsilon(r) q,
\end{aligned}
$$

where

$$
\Omega(x)=\left(\begin{array}{cc}
0 & -x^{T} \\
x & -\left[x^{\times}\right]
\end{array}\right), \forall x \in \mathbb{R}^{3},
$$

and

$$
\Upsilon(x)=\left(\begin{array}{cc}
0 & -x^{T} \\
x & {\left[x^{\times}\right]}
\end{array}\right), \forall x \in \mathbb{R}^{3} .
$$

We also consider the following linear mapping from $\mathbb{R}^{4}$ to $\mathbb{R}^{4 \times 3}$ defined as:

$$
\Xi(y)=\left(\begin{array}{c}
-\vec{y}^{T} \\
y_{0} I_{3}+\left[\vec{y}^{\times}\right]
\end{array}\right), \forall y=\left[\begin{array}{ll}
y_{0} & \vec{y}^{T}
\end{array}\right]^{T} \in \mathbb{R}^{4}
$$

It can be shown that the following relation is fulfilled [7]:

$$
\Omega(x) y=\Xi(y) x .
$$

The quaternion conjugate of $q$, denoted by $q^{-1}$ is given by:

$$
q^{-1}=\left[\begin{array}{llll}
q_{0} & -q_{1} & -q_{2} & -q_{3}
\end{array}\right]^{T} .
$$

\section{Attitude estimation as filtering of a descriptor system}

\subsection{System modeling}

The discrete-time equation of attitude kinematics is given by [2]:

$$
q_{k+1}=\left[I_{4}+\frac{\Delta t}{2} \Omega\left(\omega_{k+1}\right)\right] q_{k},
$$

where, $\omega_{k+1}$ and $\Delta t$ denote respectively the angular velocity and the sampling period.

In attitude estimation, it is usual to resort to Eq.(16) as the process model, while the following two equations constitute the observation model:

$$
\begin{aligned}
y_{a, k} & =C\left(q_{k}\right)\left(G+a_{p, k}\right)+\delta_{a, k}, \\
y_{m, k} & =C\left(q_{k}\right) m+\delta_{m, k} .
\end{aligned}
$$

The accelerometer output in Eq.(17) can be written such as $y_{a, k}=y_{a 0, k}+\delta_{a, k}$, where $y_{a 0, k}$ is the true acceleration vector. We consider the quaternion vectors $Y_{a, k}=\left[\begin{array}{ll}0 & y_{a 0, k}^{T}\end{array}\right]^{T}, \bar{G}=\left[\begin{array}{ll}0 & G^{T}\end{array}\right]^{T}$ and $\bar{a}_{p, k}=$ $\left[\begin{array}{ll}0 & a_{p, k}^{T}\end{array}\right]^{T}$ associated respectively with $y_{a 0, k}, G$ and $a_{p, k} . Y_{a, k}$ is related to $\bar{G}$ and $\bar{a}_{p, k}$ through the quaternion $q_{k}$ as follows [7]:

$$
Y_{a, k}=q_{k}^{-1} \otimes\left(\bar{G}+\bar{a}_{p, k}\right) \otimes q_{k} .
$$

Left multiplying $q_{k}$ on both sides of Eq.(19) and by applying the quaternion product according to Eqs.(9) and (10), we can write:

$$
\begin{aligned}
q_{k} \otimes Y_{a, k} & =\Omega\left(y_{a 0, k}\right) q_{k}, \\
\left(\bar{G}+\bar{a}_{p, k}\right) \otimes q_{k} & =\Upsilon\left(G+a_{p, k}\right) q_{k} .
\end{aligned}
$$

Substracting the right-hand sides of Eqs.(20) and (21) and substituting $y_{a 0, k}$ by $y_{a, k}-\delta_{a, k}$, lead to the following model [7]:

$$
0_{4 \times 1}=H_{a c c, k} q_{k}-\frac{1}{2} \Lambda\left(q_{k}\right) a_{p, k}+w_{a c c, k}^{q},
$$

where $H_{a c c, k}$ and $w_{a c c, k}^{q}$ are respectively the matrix containing the accelerometer measurements $y_{a, k}$ and the quaternion-dependent noise vector related to the accelerometer noise, given by:

$$
\begin{gathered}
H_{a c c, k}=\frac{1}{2}\left(\begin{array}{cc}
0 & -\left(y_{a, k}-G\right)^{T} \\
\left(y_{a, k}-G\right) & -\left[\left(y_{a, k}+G\right)^{\times}\right]
\end{array}\right), \\
w_{a c c, k}^{q}=-\frac{1}{2} \Xi\left(q_{k}\right) \delta_{a, k} .
\end{gathered}
$$

$\Lambda\left(q_{k}\right)$ is the matrix defined by:

$$
\Lambda\left(q_{k}\right)=\left(\begin{array}{c}
-{\overrightarrow{q_{k}}}^{T} \\
-\left[{\overrightarrow{q_{k}}}^{\times}\right]+q_{0, k} I_{3}
\end{array}\right) .
$$

Following the same steps we get the model related to the magnetometer measurements:

$$
0_{4 \times 1}=H_{m g, k} q_{k}+w_{m g, k}^{q},
$$


where $H_{m g, k}$ is a $4 \times 4$ matrix containing the magnetometer measurements $y_{m g, k}$ :

$$
H_{m g, k}=\frac{1}{2}\left(\begin{array}{cc}
0 & -\left(y_{m, k}-m\right)^{T} \\
\left(y_{m, k}-m\right) & -\left[\left(y_{m, k}+m\right)^{\times}\right]
\end{array}\right) .
$$

The quaternion-dependent noise vector $w_{m g, k}^{q}$ related to magnetometer noise is given by:

$$
w_{m g, k}^{q}=-\frac{1}{2} \Xi\left(q_{k}\right) \delta_{m, k}
$$

On the other hand, given an estimate $\hat{q}_{k}$, a gyroscopebased attitude prediction can be obtained as:

$$
q_{k+1}^{\omega}=\left[I_{4}+\frac{\Delta t}{2} \Omega\left(y_{g, k+1}\right)\right] \hat{q}_{k}
$$

From Eq.(6) and using the linearity of $\Omega($.$) , we get:$

$$
\Omega\left(y_{g, k+1}\right)=\Omega\left(\omega_{k+1}\right)+\Omega\left(\delta_{g, k+1}\right) .
$$

One can easily show that (using Eqs.(16) and (29)):

$$
q_{k+1}^{\omega}=q_{k+1}+\tilde{w}_{k+1}
$$

with $\quad \tilde{w}_{k+1}=\left[I_{4}+\frac{\Delta t}{2} \Omega\left(\omega_{k+1}\right)\right] \epsilon_{k}^{q}+\frac{\Delta t}{2} \Omega\left(\delta_{g, k+1}\right) \hat{q}_{k}$ and $\epsilon_{k}^{q}=\hat{q}_{k}-q_{k}$ is the quaternion estimation error. Using the identity defined in Eq.(14) and replacing $\hat{q}_{k}$ by $\epsilon_{k}^{q}+q_{k}$, we obtain:

$$
\begin{aligned}
\tilde{w}_{k+1}= & {\left[I_{4}+\frac{\Delta t}{2} \Omega\left(\omega_{k+1}\right)\right] \epsilon_{k}^{q}+\frac{\Delta t}{2} \Xi\left(q_{k}\right) \delta_{g, k+1} } \\
& +\frac{\Delta t}{2} \Xi\left(\epsilon_{k}^{q}\right) \delta_{g, k+1}
\end{aligned}
$$

In what follows, we derive an alternative model for attitude where $q_{k+1}^{\omega}$ (calculated from gyroscope measurements) is used as an observation while the accelerometer measurements are used to build the process model. Therefore, the unknown external acceleration will impact only the process model. Before defining the state-space model, let consider the following assumption:

Assumption III.1 The external acceleration $a_{p, k}$ is modeled as:

$$
a_{p, k+1}=a_{p, k}+\epsilon_{p, k}
$$

where $\epsilon_{p, k}$ is the modeling error assumed to be a white noise with covariance matrix $R_{p}$.

Now, we can state the following proposition:
Proposition III.2 Consider the sensors outputs $y_{g, k+1}$, $y_{a, k+1}$ and $y_{m, k+1}$ (defined in Eqs.(3)-(5)) and $q_{k+1}^{\omega}$ the predicted quaternion (pseudo-measurement) resulting from Eq.(29). Under Assumption III.1, the attitude dynamics can be represented by the following descriptor system:

$$
\begin{aligned}
H_{a c c, k+1} q_{k+1} & =H_{a c c, k} q_{k}-\tilde{a}_{p, k+1}+v_{k+1} \\
z_{k+1} & =N_{k+1} q_{k+1}+n_{k+1}
\end{aligned}
$$

where $q_{k}$ is the discrete-time quaternion, $H_{a c c, k}$ is defined in Eq.(23) and $\tilde{a}_{p, k+1} \in \mathbb{R}^{3}$ is an unkown input which depends on the external acceleration $a_{p, k}$. It is given by:

$$
\tilde{a}_{p, k+1}=-\frac{1}{2} \Lambda\left(q_{k+1}-q_{k}\right) a_{p, k}
$$

$v_{k+1}$ is the process noise defined by:

$v_{k+1}=\frac{1}{2} \Xi\left(q_{k+1}\right) \delta_{a, k+1}-\frac{1}{2} \Xi\left(q_{k}\right) \delta_{a, k}+\frac{1}{2} \Lambda\left(q_{k+1}\right) \epsilon_{p, k}$.

$z_{k+1}, \quad N_{k+1}$ and $n_{k+1}$ are respectively the observation vector, matrix and noise: $z_{k+1}=\left(\begin{array}{l}q_{k+1}^{\omega} \\ 0_{4 \times 1}\end{array}\right)$, $N_{k+1}=\left(\begin{array}{c}I_{4} \\ H_{m g, k+1}\end{array}\right), n_{k+1}=\left(\begin{array}{c}\tilde{w}_{k+1} \\ w_{m g, k+1}^{q}\end{array}\right)$.

Proof. We consider Eq.(22) written for two consecutive time-samples $k$ and $k+1$. By substracting the two equations and taking the difference $\epsilon_{p, k}$, we directly get Eq.(33) where $v_{k+1}$ and $\tilde{a}_{p, k+1}$ are respectively the process noise (defined in Eq.(36)) and the unknown input (Eq.(35)).

The first observation vector is defined in Eq.(30) while the second observation vector is related to the magnetometer measurments according to Eq.(26). Therefore, Eqs.(33) and (34) define a descriptor system since by construction $\operatorname{rank}\left(H_{a c c, k+1}\right)=2$, i.e. $H_{a c c, k+1}$ is rank deficient.

The term $\tilde{a}_{p, k+1}$ is unknown and needs to be estimated. By extending the state of the system, the state-space model in Eqs.(33) and (34) can be rewritten as follows:

$$
\begin{aligned}
E_{k+1} x_{k+1} & =A_{k} x_{k}+v_{k+1}, \\
z_{k+1} & =\bar{N}_{k+1} x_{k+1}+n_{k+1}
\end{aligned}
$$

where $\quad x_{k+1}=\left(\begin{array}{c}q_{k+1} \\ \tilde{a}_{p, k+1}\end{array}\right), E_{k+1}=\left[\begin{array}{ll}H_{a c c, k+1} & I_{4}\end{array}\right]$, $A_{k}=\left[\begin{array}{ll}H_{a c c, k} & 0_{4 \times 4}\end{array}\right]$ and $\bar{N}_{k+1}=\left(\begin{array}{cc}I_{4} & 0_{4 \times 4} \\ H_{m g, k+1} & 0_{4 \times 4}\end{array}\right)$. The filter design based on this state-space model (Eq.(37)), is described in what follows. We call it Quaternion Descriptor Filter (QDF). 


\subsection{Filter design}

The main idea of QDF is to estimate the state vector $x_{k+1}$ at time $k+1$, represented by $\hat{x}_{k+1}$, based on the estimate $\hat{x}_{k}$ and the observation vector $z_{k+1}$ defined in Eq.(38). First, we check the uniqueness of the solution. From [17] we know that the system defined by Eqs.(37) and (38) has a unique solution, if the matrix $\left(\begin{array}{l}E_{k+1} \\ \bar{N}_{k+1}\end{array}\right)$, has full column rank. In our case, $\operatorname{rank}\left(\begin{array}{c}\lambda E_{k+1}-A_{k} \\ \bar{N}_{k+1}\end{array}\right)=8$, by construction, i.e. the matrix pencil has full column rank. Second, we define $\epsilon_{k}=\hat{x}_{k}-x_{k}$ as the state estimation error and we rewrite Eqs.(37) and (38) as follows:

$$
y_{k+1}=F_{k+1} x_{k+1}+\beta_{k+1},
$$

where

$$
\begin{aligned}
& \text { where } \\
& y_{k+1}=\left(\begin{array}{c}
A_{k} \hat{x}_{k} \\
z_{k+1}
\end{array}\right), F_{k+1}=\left(\begin{array}{c}
E_{k+1} \\
\bar{N}_{k+1}
\end{array}\right), \text { and } \\
& \beta_{k+1}=\left(\begin{array}{c}
A_{k} \epsilon_{k}-v_{k+1} \\
n_{k+1}
\end{array}\right) .
\end{aligned}
$$

Assumption III.3 We assume that $w_{\text {acc, }}^{q}$ represents all the stochastic behaviour due to accelerometer noise. Therefore, $H_{a c c, k}$ will be considered as deterministic.

Lemma III.4 Under Assumption III.3, the covariance matrix of $\beta_{k+1}$ can be approximated by $V_{k+1}=$ $\operatorname{diag}\left(V_{a c c, k+1}, \tilde{R}_{k+1}, V_{m, k+1}\right)$, where diag(.) stands for a block diagonal matrix and $V_{a c c, k+1}, \tilde{R}_{k+1}$ and $V_{m, k+1}$ are respectively the covariance matrices corresponding to the errors $A_{k} \epsilon_{k}-v_{k+1}, \tilde{w}_{k+1}$ and $w_{m g, k+1}^{q}$.

$$
\begin{aligned}
V_{a c c, k+1} & =H_{a c c, k} P_{k}^{q} H_{a c c, k}^{T}+\frac{1}{2} \Xi\left(\hat{q}_{k}\right) R_{a} \Xi^{T}\left(\hat{q}_{k}\right) \\
& +\frac{\Delta t^{2}}{16} \Xi\left(\Omega\left(y_{g, k+1}\right) \hat{q}_{k}\right) R_{a} \Xi^{T}\left(\Omega\left(y_{g, k+1}\right) \hat{q}_{k}\right) \\
& +\frac{\Delta t^{2}}{16} \Lambda\left(\Omega\left(y_{g, k+1}\right) \hat{q}_{k}\right) R_{p} \Lambda^{T}\left(\Omega\left(y_{g, k+1}\right) \hat{q}_{k}\right) \\
& +\frac{1}{4} \Lambda\left(\hat{q}_{k}\right) R_{p} \Lambda^{T}\left(\hat{q}_{k}\right), \\
\tilde{R}_{k+1}= & {\left[I_{4}+\frac{\Delta t}{2} \Omega\left(y_{g, k+1}\right)\right] P_{k}\left[I_{4}+\frac{\Delta t}{2} \Omega\left(y_{g, k+1}\right)\right]^{T} } \\
+ & \left(\frac{\Delta t}{2}\right)^{2} \Xi\left(\hat{q}_{k}\right) R_{g} \Xi^{T}\left(\hat{q}_{k}\right) \\
+ & \left(\frac{\Delta t}{2}\right)^{2} \sigma_{g}^{2}\left(\operatorname{Tr}\left(P_{k}\right) I_{4}-P_{k}\right),
\end{aligned}
$$

$$
\begin{aligned}
V_{m, k+1} & =\frac{1}{4} \Xi\left(\hat{q}_{k}\right) R_{m} \Xi^{T}\left(\hat{q}_{k}\right)+ \\
& \frac{\Delta t^{2}}{16} \Xi\left(\Omega\left(y_{g, k+1}\right) \hat{q}_{k}\right) R_{m} \Xi^{T}\left(\Omega\left(y_{g, k+1}\right) \hat{q}_{k}\right),
\end{aligned}
$$

where $P_{k}^{q}$ is the covariance matrix of the quaternion estimation error $\epsilon_{k}^{q}$, and $\operatorname{Tr}(\bullet)$ denotes the matrix trace.

Proof. From the expression of $\beta_{k+1}$, we compute the covariance matrices (See the Appendix for more details) and we obtain the following results:

$$
\begin{aligned}
V_{a c c, k+1} & =H_{a c c, k} P_{k}^{q} H_{a c c, k}^{T}+\frac{1}{2} \Xi\left(q_{k}\right) R_{a} \Xi^{T}\left(q_{k}\right)+ \\
& +\frac{\Delta t^{2}}{16} \Xi\left(\Omega\left(\omega_{k+1}\right) q_{k}\right) R_{a} \Xi^{T}\left(\Omega\left(\omega_{k+1}\right) q_{k}\right) \\
& +\frac{\Delta t^{2}}{16} \Lambda\left(\Omega\left(\omega_{k+1}\right) q_{k}\right) R_{p} \Lambda^{T}\left(\Omega\left(\omega_{k+1}\right) q_{k}\right) \\
& +\frac{1}{4} \Lambda\left(q_{k}\right) R_{p} \Lambda^{T}\left(q_{k}\right), \\
\tilde{R}_{k+1}= & {\left[I_{4}+\frac{\Delta t}{2} \Omega\left(\omega_{k+1}\right)\right] P_{k}\left[I_{4}+\frac{\Delta t}{2} \Omega\left(\omega_{k+1}\right)\right]^{T} } \\
+ & \left(\frac{\Delta t}{2}\right)^{2} \Xi\left(q_{k}\right) R_{g} \Xi^{T}\left(q_{k}\right) \\
+ & \left(\frac{\Delta t}{2}\right)^{2} \sigma_{g}^{2}\left(\operatorname{Tr}\left(P_{k}\right) I_{4}-P_{k}\right) .
\end{aligned}
$$

In practice, the estimated quaternion $\hat{q}_{k}$ and the output value of gyroscope $y_{g, k+1}$ substitute respectively the actual quaternion $q_{k}$ and the true angular velocity value $\omega_{k+1}$, to compute $V_{a c c, k+1}, \tilde{R}_{k+1}$ and $V_{m, k+1}$. Also considering Assumption III.3, we get Eqs.(40)-(42).

The attitude estimation problem can be considered as a Weighted Least Square (WLS) problem which consists in finding the state $x_{k+1}$ in Eq.(39) by minimizing the following cost function:

$$
\begin{aligned}
J & =\left\|y_{k+1}-F_{k+1} x_{k+1}\right\|_{V_{k+1}}^{2} \\
& +\left(y_{k+1}-F_{k+1} x_{k+1}\right)^{T} V_{k+1}\left(y_{k+1}-F_{k+1} x_{k+1}\right)
\end{aligned}
$$

Lemma III.5 There exists a linear estimator of finite error variance of Eqs.(37) and (38) if $F_{k+1}$ has full column rank ([17]).

Proposition III.6 Given the estimate $\hat{x}_{k}$ and its corresponding error covariance matrix $P_{k}$, the WLS estimator (denoted by QDF) of the state vector, 
represented by $\hat{x}_{k+1}$, composed of the quaternion $\hat{q}_{k+1}$ and the unknown input $\hat{\tilde{a}}_{p, k+1}$, is given by:

$$
\begin{gathered}
\hat{x}_{k+1}=P_{k+1}\left(\begin{array}{c}
H_{a c c, k+1}^{T} V_{a c c, k}^{-1} H_{a c c, k} \hat{q}_{k}+\tilde{R}_{k}^{-1} q_{k+1}^{\omega} \\
V_{a c c, k}^{-1} H_{a c c, k} \hat{q}_{k}
\end{array}\right), \\
P_{k+1}=\left(\begin{array}{cc}
P_{1,1} & P_{1,2} \\
P_{2,1} & P_{2,2}
\end{array}\right)^{-1},
\end{gathered}
$$

where

$$
\begin{aligned}
P_{1,1}= & H_{a c c, k+1}^{T} V_{a c c, k}^{-1} H_{a c c, k+1}+\tilde{R}_{k}^{-1} \\
& +H_{m g, k+1}^{T} V_{m, k}^{-1} H_{m g, k+1} \\
P_{1,2}= & H_{a c c, k+1}^{T} V_{a c c, k}^{-1} \\
P_{2,1}= & V_{a c c, k}^{-1} H_{a c c, k+1} \\
P_{2,2}= & V_{a c c, k}^{-1} .
\end{aligned}
$$

Proof. We consider Eq.(39) where $F_{k+1}$ has full rank by construction (rank $\left(F_{k+1}\right)=8$ ), then, from Lemma III.5, it is well known that the WLS estimate of $x_{k+1}$ and the error covariance matrix $P_{k+1}$ are given by:

$$
\begin{aligned}
\hat{x}_{k+1} & =\left(F_{k+1}^{T} V_{k+1}^{-1} F_{k+1}\right)^{-1} F_{k+1}^{T} V_{k+1}^{-1} y_{k+1}, \\
P_{k+1} & =\left(F_{k+1}^{T} V_{k+1}^{-1} F_{k+1}\right)^{-1}
\end{aligned}
$$

When we replace $F_{k+1}, V$, and $y_{k+1}$, we get: $P_{k+1}^{-1}=\left(\begin{array}{lll}H_{a c c, k+1}^{T} & I_{4} & H_{m g, k+1}^{T} \\ I_{4} & 0_{4 \times 4} & 0_{4 \times 4}\end{array}\right) \times$

$\left(\begin{array}{ccc}V_{a c c, k}^{-1} & 0_{4 \times 4} & 0_{4 \times 4} \\ 0_{4 \times 4} & \tilde{R}_{k}^{-1} & 0_{4 \times 4} \\ 0_{4 \times 4} & 0_{4 \times 4} & V_{m, k}^{-1}\end{array}\right)\left(\begin{array}{cc}H_{a c c, k+1} & I_{4} \\ I_{4} & 0_{4 \times 4} \\ H_{m g, k+1} & 0_{4 \times 4}\end{array}\right)$ $\hat{x}_{k+1}=P_{k+1} \times$

$\left(\begin{array}{ccc}H_{a c c, k+1}^{T} V_{a c c, k}^{-1} & \tilde{R}_{k}^{-1} & H_{m g, k+1} V_{m, k}^{-1} \\ V_{a c c, k}^{-1} & 0_{4 \times 4} & 0_{4 \times 4}\end{array}\right)\left(\begin{array}{c}A_{k} \hat{x}_{k} \\ q_{k+1}^{\omega} \\ 0_{4 \times 1}\end{array}\right)$.

Finally, we do a simple matrix multiplication and invert $P_{k+1}^{-1}$, to get $\hat{x}_{k+1}$ and $P_{k+1}$ as given in Eqs.(44) and (45).

Remark III.7 To ensure a unit norm quaternion at each step, a normalization step $\hat{q}_{k+1}=\frac{\hat{q}_{k+1}}{\left\|\hat{q}_{k+1}\right\|}$ is introduced.

\section{Simulation results}

In this section, we evaluate the filter performance by means of numerical simulations and provide a comparative evaluation with methods in the literature.

For this purpose, the sensors data is generated and sampled with a period $\Delta t=0.01 \mathrm{~s}$. We consider a $3 \mathrm{D}$
Table 1. Angular velocity scenario

\begin{tabular}{ccc}
\hline \hline Angular rate & $0<k \Delta t \leq 50 s$ & $50 s<k \Delta t \leq 100 s$ \\
\hline$\omega_{x, k}$ & $0.2 \cos (1.5 k \Delta t)$ & $-0.9 \sin (1.2 k \Delta t)$ \\
\hline$\omega_{y, k}$ & $0.3 \sin (0.9 k \Delta t)$ & $0.4 \cos (-0.5 k \Delta t)$ \\
\hline$\omega_{z, k}$ & $0.05 \cos (1.2 k \Delta t)$ & $0.9 \sin (2.5 k \Delta t)$ \\
\hline
\end{tabular}

rigid body motion scenario with the angular velocity given in Table 1. A reference sequence of quaternions $q_{k}$ is generated during $100 s$ using the angular velocity in Table 1 and the discrete-time kinematic equation (16). The accelerometer and magnetometer measurements are generated using Eqs.(4) and (5), respectively, and the rotation matrix in Eq.(2) is computed using the quaternion $q_{k}$. After that, a random zero-mean white noise is added to each sensor output to represent the imperfections according to Eqs.(3)-(5). Standard deviation of noises is chosen as follows: $\sigma_{a}=$ $0.02 \mathrm{~m} / \mathrm{s}^{2}$ for accelerometer, $\sigma_{m}=0.05$ Gauss for magnetometer and $\sigma_{g}=0.05 \mathrm{rad} / \mathrm{s}$ for gyroscope. The proposed simulation is achieved under near realistic accelerations similar to those observed during experimental tests in Section V. The external acceleration $a_{p, k}$ is added to the accelerometer measurements according to Eq.(4). It was randomly generated as follows: $a_{p, k}=0.8 a_{r e f}, 1.5 a_{r e f}, 0.1 a_{r e f}$ if $k \in I_{1}, I_{2}, I_{3}$, respectively. Here $a_{\text {ref }} \in \mathbb{R}^{3}$ is a random vector, $I_{1}=$ $\left[\begin{array}{ll}4.3 & 11 s\end{array}\right] \cup\left[\begin{array}{ll}15 & 17.5 s\end{array}\right] \cup\left[\begin{array}{ll}60 & 80 s\end{array}\right], \quad I_{2}=\left[\begin{array}{ll}26 & 36 s\end{array}\right]$ and $I_{3}=\left[\begin{array}{ll}20 & 23 s\end{array}\right] \cup\left[\begin{array}{ll}42 & 47 s\end{array}\right]$. The covariance matrix $R_{p}$ in Eq.(32) is given by: $R_{p}=\sigma_{p}^{2} I_{3}$, where $\sigma_{p}$ is the standard deviation, empirically tuned as $\sigma_{p}=0.05 \mathrm{~m} / \mathrm{s}^{2}$.

\subsection{Evaluation of the QDF performance and comparative analysis}

In order to evaluate the filter performance, we plot the trace of the rotation matrix of estimation error trace $\left(I_{3}-C(\hat{q})^{T} C(q)\right.$ in Fig. 1. This figure shows a comparaison of the QDF with methods commonly used in practice: the Quaternion Kalman Filter (QKF) [7], the Gradient Descent Algorithm (GDA) [4] and the Complementary Filter (CF) [9]. All filters are initialized with the same attitude value $\hat{q}(0)=\left[\begin{array}{llll}1 & 0 & 0 & 0\end{array}\right]^{T}$. The observation vector $q_{k}^{\omega}$ in Eq.(30) is initialized with $q_{0}^{\omega}=\left[\begin{array}{llll}-0.2911 & 0.6002 & -0.7353 & -0.1195\end{array}\right]^{T}$ and the initial estimation error covariance matrix is set to $P_{0}=0.1 I_{8}$. For $\mathrm{QKF}$, the initial attitude covariance matrix is set to $P(0)=0.1 I_{4}$. To be able to perform a fair comparison, the parameters of these estimators are tuned according to a practically common criteria. 

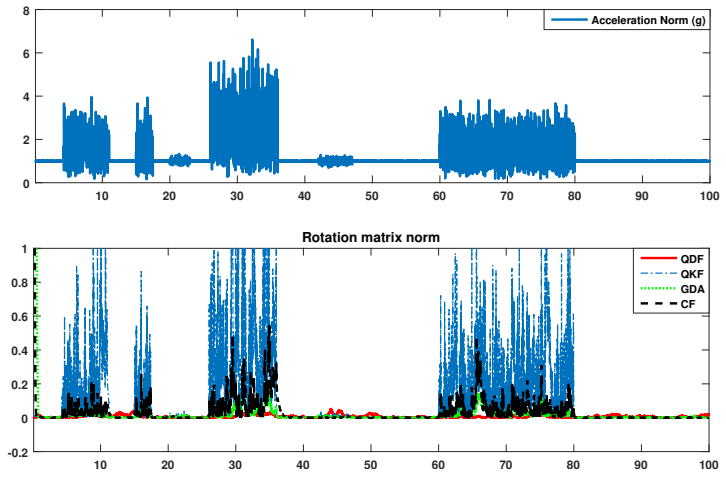

Fig. 1. Attitude estimation errors

Table 2. RMSE of Euler angles for the estimators CF, GDA, QDF, QKF

\begin{tabular}{cccc}
\hline \hline & Roll $\left({ }^{\circ}\right)$ & Pitch $\left({ }^{\circ}\right)$ & Yaw $\left({ }^{\circ}\right)$ \\
\hline CF & 23,5174 & 6,6012 & 35,9623 \\
\hline GDA & 20,7900 & 8,0620 & 30,6315 \\
\hline QKF & 21,8379 & 5,9015 & 30,3436 \\
\hline QDF & 0,9827 & 1,4433 & 2,0687 \\
\hline
\end{tabular}

The gain $\beta$ in the GDA [4] was fixed to 2 while the gain $K_{p}$ in the CF [9] was fixed to 10. Recall that for both QKF and QDF, the gains are tuned automatically. As we can see, the proposed filter provides the best estimation even when external acceleration appears for a long period and with a high magnitude, while the other filters lose accuracy in dynamic phases (for example between $23 s$ and $37 s$ ). In addition, we evaluate the performance of the approaches cited above using the Root Mean Square Error (RMSE) of Euler angles as: $R M S E=\sqrt{\frac{1}{T} \sum_{t=0}^{T} x_{\text {angle }}^{2}(t)}$, where $T$ stands for the time interval and $x_{\text {angle }}$, angle $\in\{$ pitch, roll, yaw $\}$ is the computed error between the estimated angle and the reference one. From Table 2, it is clear that QDF outperforms the other estimators in the high acceleration scenario and provides the best results in terms of RMS attitude estimation errors. Indeed, in the low-acceleration scenario, our approach performs at least as good as the other methods since the accelerometer output is the projection of $G$. However, when the external acceleration appears, the estimators GDA, CF and QKF lose accuracy since the assumption on external acceleration is no longer fulfilled. In conclusion, we want to stress that these approaches are not really designed to deal with the external acceleration problem and assume that the accelerometer measurements represent only the projection of $G$.
However, these methods are, nowadays, commonly used in practice.

\section{Experimental results}

To assess the efficiency of QDF, several experiments were performed in the case of pedestrian navigation for different scenarios. We used the footmounted MTi-IMU developed by Xsens Technologies [21] to collect measurements. The unit sampled sensor data for an orthogonally oriented triaxial accelerometer, a triaxial gyroscope, and a triaxial magnetometer at $100 \mathrm{~Hz}$. A set of experiments was carried out at the MOCA platform based in GIPSA-Lab at University of Grenoble Alpes to collect data for different users with different weights. The foot attitude is calculated by a Vicon motion capture system, containing 12 cameras T40s, through Tracker software. Vicon reconstructs the position and orientation of objects with passive markers that reflect light sent by the spotlight. The triad composed of markers is aligned with the one of MTis sensors to synchronize later the QDF and the Vicon system. Fig. 2 shows the overall experimental setup. All subjects were asked to walk on a path marked on the room, including rectangle shaped walking with a width of $2 \mathrm{~m}$ and a length of $3 \mathrm{~m}$ during $3 \mathrm{~min}$ as in Fig. 2.

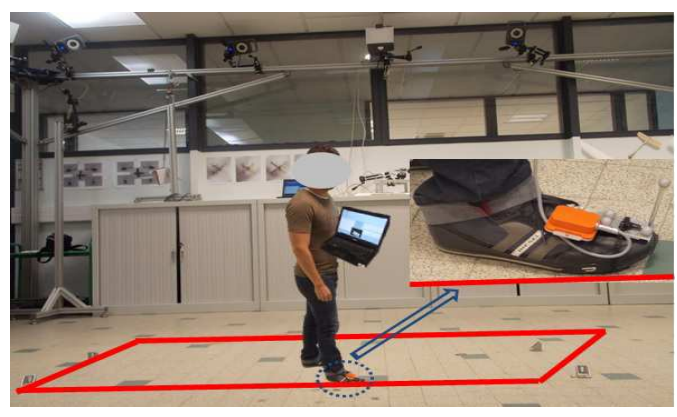

Fig. 2. Experimental setup with MTi and Vicon system

The collected data from the MTi are processed offline using the QDF implemented under Matlab to estimate the attitude (in terms of quaternion) and are compared with the quaternion calculated directly by the Vicon system which is considered as the reference. The experiments are conducted with initial conditions sufficiently far of the reference angular position and with high accelerations. Similar results are obtained with all subjects for the same experiments. Then, we represent the estimation results in Fig.3 where the external acceleration (top of the figure) and Euler angles estimation errors are shown. As we can see in this figure, although many high jumps in the external 
acceleration are observed, the attitude estimation errors doesnt increase significantly and the peaks are not so significant (except a few related to the error on the quaternion calculated by the Vicon). In fact, after each jump in the true external acceleration, we can observe that the transient time on the attitude convergence is so quick and negligible. After deep analysis, we remarked also that the sudden jump (reccurent) of errors (especially on roll and pitch) between $0^{\circ}$ and $5^{\circ}$ is related to the saturation in the gyroscope measurements (out of range of the sensor).
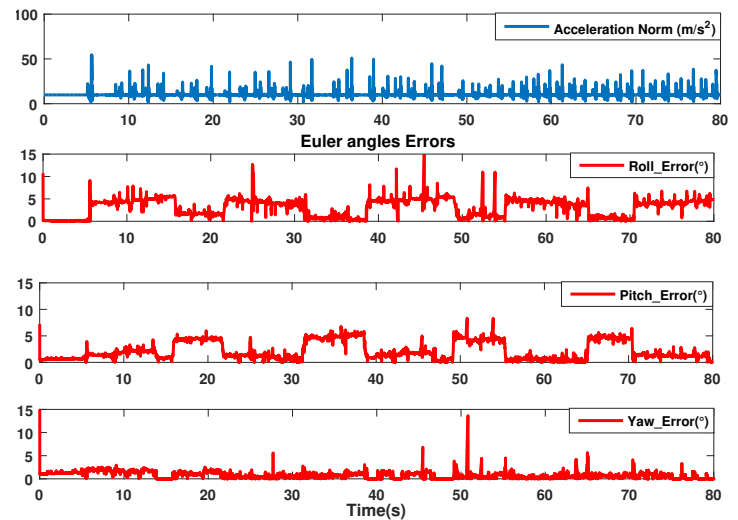

Fig. 3. External acceleration (top of the figure) and Euler angles estimation errors during experiment

By exploiting the complementary properties of all sensors (accelerometer, magnetometer and gyroscope) as explained in [9], the gyroscope and magnetometer measurements are used to compensate for errors caused by the external acceleration (in the process model). More precisely, the peaks that normally appears on attitude estimation using accelerometer measurements are more or less smoothed, with a very short transient time, by attitude observations coming from gyroscope and magnetometer measurements. Moreover, the same comparative study considered in simulation tests (section IV) has been carried out with experimental data. Then, the QDF is compared to the QKF, the GDA and the CF estimators. We plotted in Fig. 4 the Cumulative Distribution Function of the estimation errors for the three Euler angles using QDF, QKF, GDA and CF. We can note that, the QDF gives better performances than other filters. Indeed, the probability to obtain an estimation error less than $5^{\circ}$ is more than $90 \%$ for the roll, pitch and yaw angles with our approach while it is about $60 \%$ (for the roll), $79 \%$ (for the pitch) and $40 \%$ (for the yaw) with the GDA, for example.
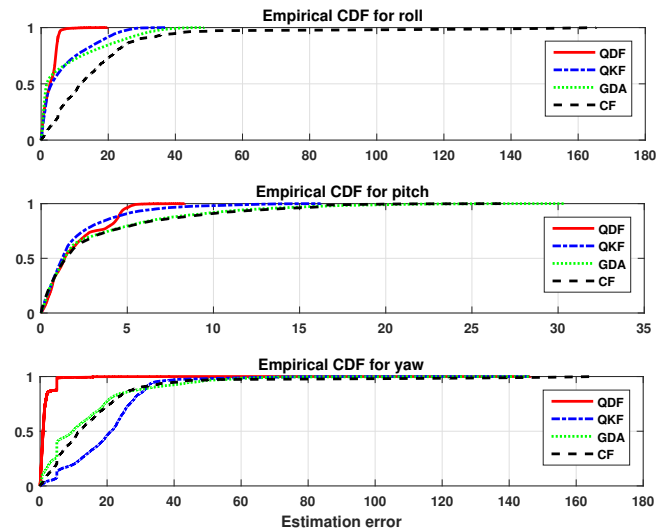

Fig. 4. Empirical Cumulative Distribution Function for Euler angles estimation errors

\section{Conclusion}

In this paper, we have introduced a descriptor model in order to carry out an attitude estimation using MARG sensors under external acceleration. The design of a Quaternion Descriptor Filter (QDF) for rigid body attitude estimation using inertial/magnetic sensors is provided with the following major goal: a new formulation of dynamical model for attitude estimation in order to produce an accurate attitude even under high external acceleration. The estimation performance is validated through numerical simulations and first experimental tests. The main feature of the proposed approach is that the process model depends on accelerometer measurements and the observation model depends on gyroscope and magnetometer measurements. This feature is particularly interesting in order to use in intermittent way the gyroscope, whose energy consumption is significant.

\section{Appendix}

Computation of the covariance matrix $V$

As stated in Section III, $V_{k+1}=$ $\operatorname{diag}\left(V_{a c c, k+1}, \tilde{R}_{k+1}, V_{m, k+1}\right)$ is the covariance matrix of the error vector $\beta_{k+1}$. In what follows we focus on the computation of $V_{a c c, k+1}, \tilde{R}_{k+1}$ and $V_{m, k+1}$.

- Computation of $V_{a c c, k+1}$ :

$V_{a c c, k+1}$ is the covariance matrix of the noise vector $A_{k} \epsilon_{k}-v_{k+1}$. It can be defined such as:

$$
\begin{aligned}
& V_{a c c, k+1}=E\left[\left(A_{k} \epsilon_{k}-v_{k+1}-E\left[A_{k} \epsilon_{k}-v_{k+1}\right]\right)\right. \\
& \left.\left(A_{k} \epsilon_{k}-v_{k+1}-E\left[A_{k} \epsilon_{k}-v_{k+1}\right]\right)^{T}\right]
\end{aligned}
$$


where $E[\bullet]$ denotes the expectation operator.

Since $E\left[v_{k+1}\right]=0$ then:

$$
\begin{aligned}
& V_{a c c, k+1}=E\left[A_{k} \epsilon_{k} \epsilon_{k}^{T} A_{k}^{T}\right]+E\left[v_{k+1} v_{k+1}^{T}\right] \\
& +2 E\left[A_{k} \epsilon_{k} v_{k+1}^{T}\right]-E\left[A_{k} \epsilon_{k}\right] E\left[A_{k} \epsilon_{k}\right]^{T} .
\end{aligned}
$$

Substituting $v_{k+1}$ by its expression as in Eq.(36), we obtain:

$$
\begin{aligned}
& V_{a c c, k+1}=\frac{1}{4} E\left[\Xi\left(q_{k+1}\right) \delta_{a, k+1} \delta_{a, k+1}^{T} \Xi^{T}\left(q_{k+1}\right)\right] \\
& +E\left[A_{k} \epsilon_{k} \epsilon_{k}^{T} A_{k}^{T}\right]+\frac{1}{4} E\left[\Xi\left(q_{k}\right) \delta_{a, k} \delta_{a, k}^{T} \Xi^{T}\left(q_{k}\right)\right] \\
& +\frac{1}{4} E\left[\Lambda\left(q_{k+1}\right) \epsilon_{p, k+1} \epsilon_{p, k+1}^{T} \Lambda^{T}\left(q_{k+1}\right)\right] \\
& +E\left[A_{k} \epsilon_{k}\left(\delta_{a, k+1}^{T} \Xi^{T}\left(q_{k+1}\right)-\delta_{a, k}^{T} \Xi^{T}\left(q_{k}\right)\right)\right] \\
& -E\left[A_{k} \epsilon_{k}\right] E\left[A_{k} \epsilon_{k}\right]^{T}+E\left[A_{k} \epsilon_{k} \epsilon_{p, k+1}^{T} \Lambda^{T}\left(q_{k+1}\right)\right] .
\end{aligned}
$$

Taking into account the unbiasedness of the estimator $\left(E\left[\epsilon_{k}\right]=0\right)$ and that $A_{k}$ is approximated by a deterministic matrix (Assumption III.3), we get:

$$
\begin{aligned}
& V_{a c c, k+1}=\frac{1}{4} E\left[\Xi\left(q_{k+1}\right) \delta_{a, k+1} \delta_{a, k+1}^{T} \Xi^{T}\left(q_{k+1}\right)\right] \\
& +\frac{1}{4} E\left[\Xi\left(q_{k}\right) \delta_{a, k} \delta_{a, k}^{T} \Xi^{T}\left(q_{k}\right)\right]+A_{k} P_{k} A_{k}^{T} \\
& +\frac{1}{4} E\left[\Lambda\left(q_{k+1}\right) \epsilon_{p, k+1} \epsilon_{p, k+1}^{T} \Lambda^{T}\left(q_{k+1}\right)\right] \\
& +A_{k} E\left[\epsilon_{k}\left(\delta_{a, k+1}^{T} \Xi^{T}\left(q_{k+1}\right)-\delta_{a, k}^{T} \Xi^{T}\left(q_{k}\right)\right)\right] \\
& +A_{k} E\left[\epsilon_{k} \epsilon_{p, k+1}^{T} \Lambda^{T}\left(q_{k+1}\right)\right] .
\end{aligned}
$$

Since $\epsilon_{k}, \epsilon_{p, k+1}$ and $\delta_{a, k}$ are independent, then: $E\left[A_{k} \epsilon_{k}\left(\delta_{a, k+1}^{T} \Xi^{T}\left(q_{k+1}\right)-\delta_{a, k}^{T} \Xi^{T}\left(q_{k}\right)\right)\right]=0 \quad$ and $E\left[A_{k} \epsilon_{k} \epsilon_{p, k}^{T} \Lambda^{T}\left(q_{k+1}\right)\right]=0$.

Now, we compute $E\left[\Xi\left(q_{k+1}\right) \delta_{a, k+1} \delta_{a, k+1}^{T} \Xi^{T}\left(q_{k+1}\right)\right]$ :

$$
\begin{aligned}
& E\left[\Xi\left(q_{k+1}\right) \delta_{a, k+1} \delta_{a, k+1}^{T} \Xi^{T}\left(q_{k+1}\right)\right]= \\
& E\left[\Xi\left(q_{k}+q_{\alpha}\right) \delta_{a, k+1} \delta_{a, k+1}^{T} \Xi^{T}\left(q_{k}+q_{\alpha}\right)\right]= \\
& E\left[\left(\Xi\left(q_{k}\right)+\Xi\left(q_{\alpha}\right)\right) \delta_{a, k+1} \delta_{a, k+1}^{T}\left(\Xi^{T}\left(q_{k}\right)+\Xi^{T}\left(q_{\alpha}\right)\right)\right]= \\
& E\left[\Xi\left(q_{k}\right) \delta_{a, k+1} \delta_{a, k+1}^{T} \Xi^{T}\left(q_{k}\right)\right]+ \\
& E\left[\Xi\left(q_{\alpha}\right) \delta_{a, k+1} \delta_{a, k+1}^{T} \Xi^{T}\left(q_{\alpha}\right)\right] .
\end{aligned}
$$

Here, $q_{\alpha}$ is the difference between two consecutive quaternions $q_{k+1}$ and $q_{k}$, then, $q_{\alpha}=\frac{\Delta t}{2} \Omega\left(\omega_{k+1}\right) q_{k}$. From Assumption II.2, we have $E\left[\delta_{a, k+1} \delta_{a, k+1}^{T}\right]=R_{a}$, then:

$$
\begin{aligned}
& E\left[\Xi\left(q_{k+1}\right) \delta_{a, k+1} \delta_{a, k+1}^{T} \Xi^{T}\left(q_{k+1}\right)\right]= \\
& \Xi\left(q_{k}\right) R_{a} \Xi^{T}\left(q_{k}\right)+\Xi\left(q_{\alpha}\right) R_{a} \Xi^{T}\left(q_{\alpha}\right) .
\end{aligned}
$$

In the same way, we get the expression of $\frac{1}{4} E\left[\Lambda\left(q_{k+1}\right) \epsilon_{p, k+1} \epsilon_{p, k+1}^{T} \Lambda^{T}\left(q_{k+1}\right)\right]$ :

$$
\begin{aligned}
& \frac{1}{4} E\left[\Lambda\left(q_{k+1}\right) \epsilon_{p, k} \epsilon_{p, k}^{T} \Lambda^{T}\left(q_{k+1}\right)\right]= \\
& \frac{1}{4} \Lambda\left(q_{k}\right) R_{p} \Lambda^{T}\left(q_{k}\right)+\frac{1}{4} \Lambda\left(q_{\alpha}\right) R_{p} \Lambda^{T}\left(q_{\alpha}\right) .
\end{aligned}
$$

Finally, we substitute $A_{k}$ by its expression $\left[\begin{array}{ll}H_{a c c, k} & 0_{4 \times 4}\end{array}\right]$ and $q_{\alpha}$ by $\frac{\Delta t}{2} \Omega\left(\omega_{k+1}\right) q_{k}$, in Eqs.(5153), then we get $V_{a c c, k}$ as:

$$
\begin{aligned}
& V_{a c c, k}=H_{a c c, k} P_{k}^{q} H_{a c c, k}^{T} \\
& +\frac{1}{2} \Xi\left(q_{k}\right) R_{a} \Xi^{T}\left(q_{k}\right)+\frac{1}{4} \Lambda\left(q_{k}\right) R_{p} \Lambda^{T}\left(q_{k}\right) \\
& +\frac{\Delta t^{2}}{16} \Xi\left(\Omega\left(\omega_{k+1}\right) q_{k}\right) R_{a} \Xi^{T}\left(\Omega\left(\omega_{k+1}\right) q_{k}\right) \\
& +\frac{\Delta t^{2}}{16} \Lambda\left(\Omega\left(\omega_{k+1}\right) q_{k}\right) R_{p} \Lambda^{T}\left(\Omega\left(\omega_{k+1}\right) q_{k}\right) .
\end{aligned}
$$

- Computation of $\tilde{R}_{k+1}$ :

$\tilde{R}_{k+1}$ is the covariance matrix of the error $\tilde{w}_{k+1}$ defined in Eq.(30). Then,

$$
\tilde{R}_{k+1}=E\left[\left(\tilde{w}_{k+1}-E\left[\tilde{w}_{k+1}\right]\right)\left(\tilde{w}_{k+1}-E\left[\tilde{w}_{k+1}\right]\right)^{T}\right] .
$$

Since $E\left[\epsilon_{k}^{q}\right]=0$ (unbiased estimator), then $E\left[\tilde{w}_{k+1}\right]=$ 0 and:

$$
\tilde{R}_{k+1}=E\left[\tilde{w}_{k+1} \tilde{w}_{k+1}^{T}\right] .
$$

Replacing $\tilde{w}_{k+1}$ by its expression $\left[I_{4}+\right.$ $\left.\frac{\Delta t}{2} \Omega\left(\omega_{k+1}\right)\right] \epsilon_{k}^{q}+\frac{\Delta t}{2} \Xi\left(\hat{q}_{k}\right) \delta_{g, k+1}$ and taking into account the independance of $\delta_{g, k+1}$ and $\epsilon_{k}^{q}$, we get:

$$
\begin{aligned}
& \tilde{R}_{k+1}=\left[I_{4}+\frac{\Delta t}{2} \Omega\left(\omega_{k+1}\right)\right] E\left[\epsilon_{k}^{q}\left(\epsilon_{k}^{q}\right)^{T}\right]\left[I_{4}+\frac{\Delta t}{2} \Omega\left(\omega_{k+1}\right)\right] \\
& +\left(\frac{\Delta t}{2}\right)^{2} \Xi\left(q_{k}\right) E\left[\delta_{g, k+1} \delta_{g, k+1}^{T}\right] \Xi^{T}\left(q_{k}\right) \\
& +\left(\frac{\Delta t}{2}\right)^{2} E\left[\Xi\left(\epsilon_{k}^{q}\right) \delta_{g, k+1} \delta_{g, k+1}^{T} \Xi^{T}\left(\epsilon_{k}^{q}\right)\right] .
\end{aligned}
$$

Denote by $B=E\left[\Xi\left(\epsilon_{k}^{q}\right) \delta_{g, k+1} \delta_{g, k+1}^{T} \Xi^{T}\left(\epsilon_{k}^{q}\right)\right]$, $\left[\begin{array}{lll}d_{1} & d_{2} & d_{3}\end{array}\right]^{T}=\delta_{g, k+1}$ and $\left[\begin{array}{llll}e_{0} & e_{1} & e_{2} & e_{3}\end{array}\right]^{T}=\epsilon_{k}^{q}$, we compute the matrix $B$ and we obtain:

$$
\begin{aligned}
B & =\sigma_{g}^{2} \times \\
& \left(\begin{array}{cccc}
e_{1}^{2}+e_{2}^{2}+e_{3}^{2} & -e_{0} e_{1} & -e_{0} e_{2} & -e_{0} e_{3} \\
-e_{0} e_{1} & e_{0}^{2}+e_{2}^{2}+e_{3}^{2} & -e_{1} e_{2} & -e_{0} e_{3} \\
--e_{0} e_{2} & -e_{1} e_{2} & e_{0}^{2}+e_{1}^{2}+e_{3}^{2} & -e_{0} e_{3} \\
-e_{0} e_{3} & -e_{1} e_{3} & -e_{2} e_{3} & e_{0}^{2}+e_{1}^{2}+e_{2}^{2}
\end{array}\right) \\
& =\sigma_{g}^{2}\left(\operatorname{Tr}\left(E\left[\epsilon_{k}^{q}\left(\epsilon_{k}^{q}\right)^{T}\right]\right) I_{4}-E\left[\epsilon_{k}^{q}\left(\epsilon_{k}^{q}\right)^{T}\right]\right) \\
& =\sigma_{g}^{2}\left(\operatorname{Tr}\left(P_{k+1}^{q}\right) I_{4}-P_{k+1}^{q}\right) .
\end{aligned}
$$

with $P_{k+1}^{q}$ is the covariance matrix of the quaternion estimation error $\epsilon_{k}^{q}$. Finally, we obtain the expression of $\tilde{R}_{k+1}$ defined in Eq.(41). Also, the matrix $V_{m, k+1}$ in Eq.(42) can be obtained by following similar steps.

\section{Acknowledgment}

The experimental platform has been designed by the GIPSA-lab's technical staff and partially funded by EquipEx Robotex (ANR10-EQPX-44-01).

\section{REFERENCES}


1. H. Rehbinder and X. Hu, "Drift-free attitude estimation for accelerated rigid bodies," Automatica, vol. 40, no. 4, pp. 653-659, 2004.

2. F. L. Markley and J. L. Crassidis, Fundamentals of Spacecraft Attitude Determination and Control. Springer, 2014, vol. 33.

3. T. Michel, P. Genevès, H. Fourati, and N. Layaïda, "On Attitude Estimation with Smartphones," in IEEE International Conference on Pervasive Computing and Communications (PerCom), Kona, United States, Mar. 2017. [Online]. Available: https://hal.inria.fr/hal-01376745

4. S. Madgwick, A. Harrison, and R. Vaidyanathan, "Estimation of IMU and MARG orientation using a gradient descent algorithm," in proc. of IEEE Int. conf. on Rehabilitation Robotics (ICORR), 2011, pp. $1-7$.

5. A. P. Vinod, A. D. Mahindrakar, S. Bandyopadhyay, and V. Muralidharan, "A deterministic attitude estimation using a single vector information and rate gyros," IEEE/ASME Transactions on Mechatronics, vol. 20, pp. 2630-2636, 2015.

6. J. Wu, Z. Zhou, J. Chen, H. Fourati, and R. Li, "Fast Complementary Filter for Attitude Estimation Using Low-Cost MARG Sensors," IEEE Sensors Journal, , vol. 16, no. 18, pp. 69977007, Jul. 2016.

7. D. Choukroun, I. Y. Bar-Itzhack, and Y. Oshman, "Novel quaternion Kalman filter," IEEE Trans. on Aerospace and Electronic Systems, , vol. 42, no. 1, pp. 174-190, 2006.

8. R. G. Valenti, I. Dryanovski, and J. Xiao, "A linear kalman filter for MARG orientation estimation using the algebraic quaternion algorithm," IEEE Trans. Instrumentation and Measurement, , vol. 65, no. 2, pp. 467-481, 2016.

9. R. Mahony, T. Hamel, and J.-M. Pflimlin, "Nonlinear complementary filters on the special orthogonal group," IEEE Trans. on Automatic Control,, vol. 53, no. 5, pp. 1203-1218, 2008.

10. P. Marantos, Y. Koveos, and K. G. Kyriakopoulos, "UAV state estimation using adaptive complementary filters," IEEE Trans. Control Systems Technology, vol. 24, no. 4, pp. 1214-1226, 2016.

11. P. Martin and E. Salaun, "Invariant observers for attitude and heading estimation from low-cost inertial and magnetic sensors," in Proc. of IEEE Conference on Decision and Control, 2007, pp. 1039-1045.

12. P. Batista, C. Silvestre, and P. Oliveira, "Globally exponentially stable cascade observers for attitude estimation," Control Engineering Practice, vol. 20, no. 2, pp. 148-155, 2012.
13. L. Wei and W. Jinling, "Effective adaptive Kalman filter for MEMS-IMU/Magnetometers integrated attitude and heading reference systems," The Journal of Navigation, vol. 66, pp. 99-113, 2013.

14. J. K. Lee, E. Park, and S. Robinovitch, "Estimation of attitude and external acceleration using inertial sensor measurement during various dynamic conditions," IEEE Trans. on Instrumentation and Measurement,, vol. 61, no. 8, pp. 2262-2273, 2012.

15. Y. S. Suh, "Orientation estimation using a quaternion-based indirect Kalman filter with adaptive estimation of external acceleration," IEEE Trans. on Instrumentation and Measurement, vol. 59, no. 12, pp. 3296-3305, 2010.

16. H. F. Grip, T. I. Fossen, T. A. Johansen, and A. Saberi, "Globally exponentially stable attitude and gyro bias estimation with application to GNSS/INS integration," Automatica, vol. 51, pp. 158-166, 2015.

17. R. Nikoukhah, A. Willsky, and B. Levy, "Kalman filtering and riccati equations for descriptor systems," IEEE Trans. on Automatic Control, vol. 37, no. 9, pp. 1325-1342, 1992.

18. M. Darouach and L. Boutat-Baddas, "Observers for a class of nonlinear singular systems," IEEE Trans. on Automatic Control,, vol. 53, no. 11, pp. 2627-2633, 2008.

19. S. Beeby, MEMS Mechanical Sensors. Artech House, 2004.

20. NOAA. (2015) NOAA. https://www.ngdc.noaa. gov/geomag-web/.

21. X. Technologies. [Online]. Available: http: //www.xsens.com 